\title{
Impact of exercise on energy metabolism in anorexia nervosa
}

\author{
Stephan Zipfel ${ }^{1,2^{*}}$, Isabelle Mack ${ }^{1}$, Louise A Baur ${ }^{3}$, Johannes Hebebrand ${ }^{8}$, Stephen Touyz ${ }^{2,4}$, Wolfgang Herzog ${ }^{7}$, \\ Suzanne Abraham ${ }^{5,6}$, Peter SW Davies ${ }^{9}$ and Janice Russell ${ }^{2,6}$
}

\begin{abstract}
Background: Excessive physical activity is one of the most paradoxical features of anorexia nervosa (AN). However, there is individual variation in the degree of physical activity found in AN-patients. As a result, marked differences in energy expenditure may be expected. Furthermore, exercise has a positive impact on a variety of psychological disorders and the psychopathology may be different in AN displaying high exercise levels versus AN displaying low exercise levels. We analyzed the energy metabolism and psychological data in low-level exercise and high-level exercise AN-patients compared with healthy, age matched controls.

Physical activity, energy expenditure (EE) by the doubly labelled water technique and indirect calorimetry, hormone status as well as psychopathology by questionnaires for eating disorders (EDI-SC, EDI-2), eating attitude (EAT) and depression (BDI) were assessed in twelve AN patients and twelve controls.

Results: REE was decreased in AN-patients compared with controls but not when adjusted for body surface area or lean body mass. No differences in TDEE between AN- patients and controls were observed. Subgroup analyses showed that the percentage of high-level AN- exercisers was higher compared with controls. This subgroup had increased resting EE, total daily EE and scored higher on depression and the EDI-item "Drive for thinness" compared with low-level AN-exercisers.
\end{abstract}

Conclusions: We identified a significant subgroup of high-level AN-exercisers (66\%) with consecutive increased energy requirements. An easy way for clinicians to assess the amount of exercise before and in the course of treatment is a single question in the established Eating Disorder Inventory-SC (EDI-SC).

Keywords: Anorexia nervosa, Exercise, Energy expenditure, Physical activity, Doubly labelled water, Calorimetry

\section{Background}

Anorexia nervosa (AN) is a serious illness associated with a chronic course and high mortality [1]. Excessive physical activity has been mentioned by both Lasègue, 1873 [2] and Gull, 1874 [3] as one of the most paradoxical features of AN. The prevalence of hyperactivity in AN lies between 31 and $80 \%$, depending on the type of eating disorder and the study and its criteria for hyperactivity $[4,5]$. Physical activity is a metabolically expensive process. Thus, hyperactivity might be one of the underlying problems in the process of weight restoration

\footnotetext{
* Correspondence: stephan.zipfel@med.uni-tuebingen.de 'Department of Psychosomatic Medicine \& Psychotherapy, University Medical Hospital Tuebingen, Osianderstr. 5, 72074 Tuebingen, Germany ${ }^{2}$ Department of Psychological Medicine, University of Sydney, Sydney, Australia

Full list of author information is available at the end of the article
}

and maintenance. However, for clinicians it is difficult to assess physical activity due to rather accurate but timeexpensive methodologies or underreporting in selfassessment instruments $[5,6]$. Thus, often only the dietary intake is assessed resulting in false estimation of the patient's energy requirements [7]. A simple tool for clinicians to estimate the degree of physical activity would be helpful to solve this problem.

The gold standard for assessing physical activity involves the use of the doubly labelled water technique [8] which measures the total daily energy expenditure (TDEE). TDEE consists of three components: resting energy expenditure (REE), diet induced thermogenesis (DIT) and the energy cost of physical activity [9]. REE is generally reduced in emaciation due to the decreased body weight, lean body mass (LBM) and metabolic adaptations [10-12]. In contrast, TDEE, measured by doubly

\section{Biomed Central}


labelled water, is sometimes reported to be decreased in AN and sometimes to be similar to healthy controls [12]. Additionally, we have shown previously that energy metabolism is altered in the different stages of illness; for example, early refeeding of AN is accompanied with a paradoxically high DIT $[13,14]$. Fat oxidation during moderate exercise is not suppressed in AN patients where BMI $<16.5$ as it is in healthy controls [15]. Interestingly, the former studies on energy expenditure in AN did not distinguish between patients with highactivity levels and patients with low-activity levels and did not combine these measurements with assessing psychological data. Given the interindividual differences in activity of AN patients, we compared the energy metabolism, endocrine parameters as well as psychological data of AN patients with healthy controls and performed subgroup analysis by distinguishing between low-level and high-level exercisers.

\section{Methods \\ Study population}

Twelve consecutive female AN patients meeting criteria for AN according to the DSM-IV (APA 1994) which also includes the existence of amenorrhea. with $n=8(67 \%)$ from the restricting subtype and $n=4(33 \%)$ from the binge eating/purging type, were studied at the University of Sydney/Australia. Patients with comorbid physical illness or an additional axis-II disorder were excluded, as were those taking medications other than calcium supplements $(n=3)$ and oral contraceptives $(n=5)$. AN patients with comorbid depression were not excluded. Participants were instructed to continue their usual activities during the period of participation in this study.

All participants were in a nutritional rehabilitation program. Patients were asked to take part in the study not before an initial two weeks of stabilization. Initially they were encouraged to eat three meals and three snacks per day selected from a cafeteria situation and containing 4190-6280 kJ initially, increasing towards $10470-16750 \mathrm{~kJ}$ per day in the latter part of treatment. In the usual inpatient diet, $50 \%$ of the energy is derived from carbohydrate, $30 \%$ from fat and $20 \%$ from protein. Patients were fully ambulant but were required to rest on their beds for 30 minutes after breakfast and dinner and 60 minutes after lunch.

Controls $(\mathrm{N}=12)$ were healthy, post pubertal, non pregnant females and in the follicular phase of the menstrual cycle. Eating disorders, dietary restriction and over-exercise were excluded by means of a careful standardized DDE interview [16]. All participants gave informed consent to the procedure and parental consent was also obtained for subjects less than 18 years of age. The protocol for the study was approved by the Human
Ethics Committees of both Ramsay Healthcare and the University of Sydney, Australia.

\section{Protocol}

After an overnight fast, all participants (patients and controls) were first interviewed, using a structured interview [16] for the assessment of an eating disorder, including a detailed history of the exercise behaviour. They then completed structured self administered questionnaires for depression (Beck depression inventory, BDI [17]), eating disorder (Eating Disorders Inventory-Symptom Checklist, EDI-SC; Eating Disorders Inventory-II, EDI-II [18]) and eating attitude (Eating Attitudes Test, EAT-40 [19]). A fasting blood sample for the analysis of leptin, thyroid hormones and basal cortisol was collected. Body composition, REE and TDEE were assessed.

\section{Blood samples}

Fasting blood samples were immediately centrifuged and stored at $-80^{\circ} \mathrm{C}$. The radioimmunoassay (RIA) method was used to measure leptin (Mediagnost, Tuebingen, Germany; intra-assay variance: $5 \%$; interassay variance: 7.6\%) and cortisol (100T kit of the Nichols Institute Diagnostics, San Juan Capistrano, CA, USA; sensitivity: $0.8 \mu \mathrm{g} / \mathrm{l}$, intra-assay variance: $4 \%$, inter-assay variance: $8 \%)$. Triiodothyronine $\left(\mathrm{T}_{3}\right)$, thyroxine $\left(\mathrm{T}_{4}\right)$ and thyroidstimulating hormone (TSH) were measured by chemiluminiscence (ACS Centaur, Chiron Diagnostics, Fernwald, Germany; intra-assay variance: $\mathrm{T}_{3} 2.8 \%$; $\mathrm{T}_{4}$ 3.1\%; TSH 2.3\%; inter-assay variance: $\mathrm{T}_{3} 3.7 \% ; \mathrm{T}_{4} 2.7 \%$; TSH 3.6\%).

\section{Anthropometry}

Body mass index (BMI) was calculated as weight $/$ height $^{2}$ $\left(\mathrm{kg} / \mathrm{m}^{2}\right)$. Body composition was assessed via skinfold thickness by a caliper at four sites of the body. Subsequently, the body fat mass was derived from the equations of Durnin and Womersley [20]. LBM was calculated as the difference between body mass and body fat mass. Bioelectrical impedance analysis (BIA) was measured using the body composition analyzer BIA-101 (RJL system, Detroit) in order to estimate total body water.

\section{Resting energy expenditure (REE)}

A Datex Deltatrac II Metabolic Monitor (Datex, Finland) was used to measure REE over a 30 minute episode by indirect calorimetry. Participants were asked to lie still and were permitted to read or listen to music via headphones. They were prevented from sleeping.

\section{Total daily energy expenditure (TDEE)}

TDEE was measured over 15 days by the doubly labelled water method and was applied as described in detail 
elsewhere $[8,21,22]$. The amounts of labelled water taken were scaled according to estimated total body water (TBW): $\mathrm{H}_{2}^{18} \mathrm{O}, 0.25 \mathrm{~g} / \mathrm{kg}$ TBW; ${ }^{2} \mathrm{H}_{2} 0,0.1 \mathrm{~g} / \mathrm{kg} \mathrm{TBW}$ (for details see [23]) This method, which has been shown to be accurate within a range of 5 percent in adults under free living conditions, is based on the prediction of carbon dioxide production from the differential disappearance rates of two stable isotopes [8]. Energy as activity was calculated by the equation: The Physical activity level (PAL) was assessed by the equation: PAL $=$ TDEE/ REE.

\section{Data analysis}

All results are presented as mean and standard deviations (SD). The data were evaluated using SPSS for Windows. To compare group differences, student's t-tests were performed. In case of skew distribution or inhomogeneous variance, Wilcoxon 2-sample-tests were computed. Rank correlations (Spearman correlation coefficient) were used to determine the degree of relationships between TDEE and BDI scores and the items "Drive for thinness" from the EDI-2. Data were adjusted for multiple testing using the Bonferroni correction.

\section{Results}

Baseline demographic and physical characteristics of AN patients and controls are presented in Table 1. The BMI and body composition (fat mass and LBM) were significantly reduced in AN patients compared with age- matched female controls. The daily amount of exercise did not statistically differ between the groups due to the great interindividual differences as assessed by structured interview (DDE). Consequently, we differentiated between high-level exercisers and low-level exercisers by using a cutoff set at $<50 \%$ according to the EDI-SC item "What percentage of your exercise is aimed at controlling your weight?" There was a significantly higher percentage of high-level exercisers in the AN participants compared with the controls.

The amount of daily exercise in patients labeled as high-level exercisers was 3- to 4-fold higher compared with the controls and low-level exercisers, respectively. Despite the varying activity levels in the AN subgroups, no differences in BMI or LBM were observed.

In order to gain insights into the underlying energy metabolism, REE and TDEE were assessed (Table 2). REE, determined by indirect calorimetry, was significantly decreased in patients with AN compared with healthy controls (Table 2, Figure 1). However, no significant differences in REE were found between patients and controls when adjusted for body surface area or lean body mass (LBM). Interestingly, subgroup analysis revealed that the REE of high-level AN exercisers was nearly similar to the REE of healthy controls but was significantly higher than the REE of low-level AN exercisers (Table 2, Figure 2). These relationships were also observed when the REE was adjusted for LBM or body surface area.

Table 1 Baseline demographic and physical characteristics of AN patients and controls

\begin{tabular}{|c|c|c|c|c|}
\hline \multirow[t]{3}{*}{ Variable } & Anorexia nervosa (AN) & \multicolumn{2}{|c|}{ Controls } & $P$ \\
\hline & $n=12$ & \multicolumn{2}{|c|}{$n=12$} & \\
\hline & $M \pm S D$ & \multicolumn{2}{|c|}{$M \pm S D$} & \\
\hline Age (yrs.) & $21.9 \pm 6.2$ & \multicolumn{2}{|c|}{$25.3 \pm 3.5$} & n.s. \\
\hline Duration of illness (yrs.) & $4.3 \pm 3.9$ & & & \\
\hline Body mass index $\left(\mathrm{kg} / \mathrm{m}^{2}\right)$ & $15.4 \pm 1.0$ & \multicolumn{2}{|c|}{$21.8 \pm 2.5$} & $<0.001$ \\
\hline Fat mass (kg) & $6.4 \pm 2.5$ & \multicolumn{2}{|c|}{$17.9 \pm 4.5$} & $<0.001$ \\
\hline Lean body mass (kg) & $34.3 \pm 4.1$ & \multicolumn{2}{|c|}{$43.5 \pm 4.5$} & $<0.001$ \\
\hline Exercise (min/day) & $83.2 \pm 63.9$ & \multicolumn{2}{|c|}{$44.2 \pm 25.5$} & 0.06 \\
\hline Exercise intensity* & & & & 0.031 \\
\hline High intensity, n (\%) & 7 (58.3\%) & \multicolumn{2}{|c|}{$2(16.7 \%)$} & \\
\hline \multirow[t]{4}{*}{ Low intensity , n (\%) } & $5(41.7 \%)$ & \multicolumn{2}{|c|}{$10(83.3 \%)$} & \\
\hline & AN High exercisers & AN Low exercisers & Controls & \\
\hline & $\mathrm{n}=7$ & $n=5$ & $\mathrm{n}=12$ & \\
\hline & $M \pm S D$ & $M \pm S D$ & $M \pm S D$ & \\
\hline $\mathrm{BMI}\left(\mathrm{kg} / \mathrm{m}^{2}\right)$ & $15.9 \pm 1.2$ & $15.0 \pm 0.85$ & $21.8 \pm 2.5$ & $1 c, 2 c$ \\
\hline Lean body mass (kg) & $35.3 \pm 5.1$ & $33.5 \pm 3.2$ & $43.5 \pm 4.5$ & $1 c, 2 c$ \\
\hline Exercise (min/day) & $128.6 \pm 51.7$ & $33.0 \pm 23.8$ & $44.2 \pm 25.5$ & $1 c, 3 b$ \\
\hline
\end{tabular}

Values are expressed as means (M) \pm standard deviations (SD). *From EDI-SC ("What percentage of your exercise is aimed at controlling your weight $>50 \%$ cutoff"). $\mathrm{a}=P<0.05, \mathrm{~b}=P<0.01, \mathrm{c}=P<0.001$, n.s. $=$ not significant.

$1=$ AN high exercisers vs. controls, $2=$ AN low exercisers vs. controls, $3=$ AN low exercisers vs. AN high exercisers. 
Table 2 Energy expenditure of AN compared with controls

\begin{tabular}{|c|c|c|c|c|}
\hline \multirow[t]{3}{*}{ Variable } & Anorexia nervosa (AN) & \multicolumn{2}{|c|}{ Controls } & $P$ \\
\hline & $\mathrm{n}=12$ & \multicolumn{2}{|c|}{$n=12$} & \\
\hline & $M \pm S D$ & \multicolumn{2}{|c|}{$M \pm S D$} & \\
\hline Resting energy expenditure (REE, $k J / 24 h)$ & $4457.0 \pm 745.7$ & \multicolumn{2}{|c|}{$5885.5 \pm 630.3$} & $<0.001$ \\
\hline REE adjusted for body surface area, SA $\left(\mathrm{kJ}^{*} \mathrm{~m}^{-2}{ }^{*} 24^{-1}\right)$ & $3258.2 \pm 450.9$ & \multicolumn{2}{|c|}{$3475.2 \pm 195.2$} & n.s. \\
\hline REE adjusted for lean body mass, LBM $\left(\mathrm{kJ}^{*} \mathrm{~kg}^{-1} * 24 \mathrm{~h}^{-1}\right)$ & $129.9 \pm 16.5$ & \multicolumn{2}{|c|}{$135.4 \pm 6.6$} & n.s. \\
\hline \multirow[t]{4}{*}{ Total daily energy expenditure (TDEE, kJ/24h) } & $7546.1 \pm 2660.6$ & \multicolumn{2}{|c|}{$9741.1 \pm 2237.1$} & 0.077 \\
\hline & AN High exercisers & AN Low exercisers & Controls & \\
\hline & $n=7$ & $\mathrm{n}=\mathbf{5}$ & $\mathrm{n}=12$ & \\
\hline & $M \pm S D$ & $M \pm S D$ & $M \pm S D$ & \\
\hline REE (kJ/24h) & $5218 \pm 1166.9$ & $3991.5 \pm 546.8$ & $5885,4 \pm 630,3$ & $2 c, 3 a$, \\
\hline REE adjusted for $S A\left(k^{\prime *} m^{-2} * 24^{-1}\right)$ & $3761.8 \pm 479.1$ & $2968.4 \pm 318.3$ & $3475.2 \pm 195.2$ & $2 c, 3 b$ \\
\hline REE adjusted for LBM $\left(k^{*} * g^{-1} * 24 h^{-1}\right)$ & $146.0 \pm 13.7$ & $119.7 \pm 12.6$ & $135.4 \pm 6.6$ & $2 b, 3 b$ \\
\hline TDEE (kJ/24h) & $8706.9 \pm 1875.7$ & $4619,2 \pm 946.7$ & $9741.1 \pm 2237.1$ & $2 c, 3 a$, \\
\hline
\end{tabular}

Values are expressed as means $(M) \pm$ standard deviations (SD). ${ }^{2}$ REE calculated according to the Harris-Benedict equation. $\mathrm{a}=\mathrm{P}<0.05, \mathrm{~b}=\mathrm{P}<0.01, \mathrm{c}=\mathrm{P}<0.001$, n.s. = not significant.

$1=$ AN high exercisers vs. controls, $2=$ AN low exercisers vs. controls, $3=$ AN low exercisers vs. AN high exercisers.

There was no difference in TDEE between patients with AN and controls. TDEE was also comparable in high-level AN exercisers versus controls. In contrast, the low-level AN exercisers had significantly decreased TDEE values compared to the high-level exerciser subgroup and the controls, respectively (Figure 2). The physical activity level (PAL) was 1.65, in both high-level AN exercisers and controls, whereas in low-level AN exercisers the PAL was 1.16. From the restricting subtype $n=5$ patients were in the low and $n=3$ in the high-level exerciser group. In the binge

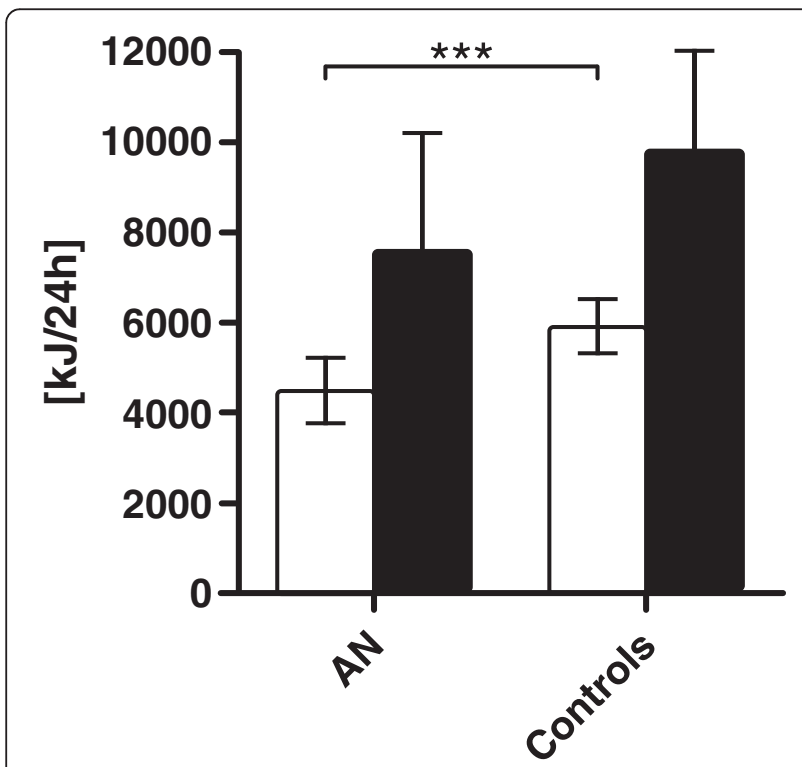

Figure 1 Resting energy expenditure (REE, white columns) and total daily energy expenditure (TDEE, black columns) in anorexia nervosa $(n=12)$ and healthy controls $(n=12)$. eating/purging subgroup all $n=4$ patients were in the highexercise group. Next, we analyzed the plasma levels for a selected panel of hormones known to be involved in energy metabolism, including TSH, $\mathrm{T}_{3}, \mathrm{~T}_{4}$, leptin and cortisol (Table 3 ). Leptin, $T_{3}$ and $T_{4}$ plasma levels were significantly reduced in patients with AN compared to the controls. A

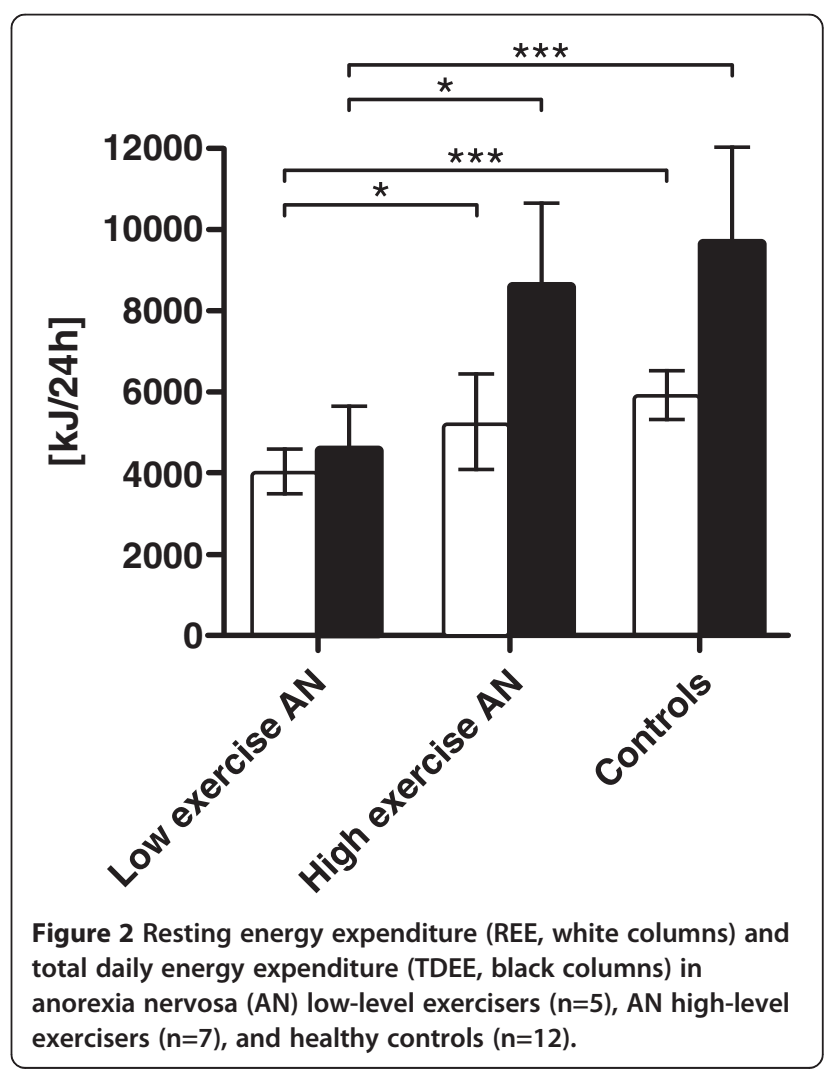


differential regulation was observed for $\mathrm{T}_{4}$, which was significantly reduced in high-level exercisers versus the controls. TSH and cortisol levels remained unchanged between the groups. For the subgroup analysis (low- versus highlevel AN exercisers), no significant differences were observed. We were not able to detect a significant association between leptin levels and TDEE (partial correlation controlled for body mass index $\mathrm{r}=-0.53, \mathrm{p}=0.28$ ).

Finally, we assessed psychological data of our study group by standardized and established questionnaires for depression (BDI), eating disorder (EDI-2) and eating attitude (EAT; Table 4). AN patients scored significantly higher on depression and on every subscale of the EAT and EDI-2, with the exception of the scale "maturity fears". Additionally, the "drive for thinness" and the degree of depression were higher in the high-level AN exercisers compared with the low-level AN exercisers. The "drive for thinness" significantly correlated with TDEE $(\mathrm{r}=0.86, \mathrm{p}<0.01)$. Moreover, in AN patients, TDEE showed a positive correlation with BDI scores $(r=0.821$, $\mathrm{p}<0.01)$.

\section{Discussion}

This energy expenditure study provides new insights into the energy metabolism of patients with AN. Here we assessed, for the first time in low-level exercise and high-level exercise AN patients, compared with healthy controls 1) the differences in their energy expenditure by analyzing TDEE and REE by doubly labeled water and indirect calorimetry, respectively, 2) their hormonal status combined with 3) psychological data for depression, eating attitudes and eating disorder by established standardized questionnaires (BDI, EAT, EDI-SC, EDI-2).
We found that the daily amount of exercise in AN patients and controls was similar despite a marked difference in weight and body composition. In accordance, using the doubly labeled water method, we found no significant differences in mean TDEE between AN patients and controls. Applying the same technique, some other studies support our findings [24-26], whereas others report a reduced TDEE in AN patients [23,27,28]. We and others have found a significant reduction in REE in patients with AN compared with healthy controls $[12,23,24,26,27]$. This observation is mainly due to the decreased amount of LBM since we and others [23,29] found no significant differences in REE when adjusted for LBM. Besides LBM, an altered thyroid metabolism influences the REE [12,30]. In this study, TSH values were similar in AN patients and controls whereas $T_{3}$ and $\mathrm{T}_{4}$ were decreased. Thus, this study supports the notion that the down regulation of the "thyrostat" in order to conserve energy is achieved by peripheral adaptive process rather than compensatory hypothalamic pituitary reaction $[10,11,30]$. We were not able to detect a significant association between TDEE and leptin levels. This finding is partly in contrast to other studies [31-33] and may be due to our limited sample size, and the fact that our patient sample demonstrated a heterogeneous sample of under/normal - and over exercisers. However, Holtkamp et al., 2006 [32] also showed an inverted U shape association so that the authors concluded that AN hyperactivity related to hypoleptinemia cannot be conceptualized as a single mode of behavior.

Next, we performed subgroup analyses due to the marked interindividual differences in the patients regarding REE, TDEE and physical activity. Since the EDI-

Table 3 Comparison of hormonal levels between AN patients and controls

\begin{tabular}{|c|c|c|c|c|}
\hline \multirow[t]{3}{*}{ Variable } & Anorexia nervosa (AN) & \multicolumn{2}{|c|}{ Controls } & $P$ \\
\hline & $n=12$ & \multicolumn{2}{|c|}{$\mathrm{n}=12$} & \\
\hline & $M \pm S D$ & \multicolumn{2}{|c|}{$M \pm S D$} & \\
\hline Leptin $(\mu \mathrm{g} / \mathrm{L})$ & $1.3 \pm 1.8$ & \multicolumn{2}{|c|}{$9.6 \pm 4.7$} & $<0.001$ \\
\hline Cortisol $(\mu \mathrm{g} / \mathrm{L})$ & $206.6 \pm 52.9$ & \multicolumn{2}{|c|}{$219.6 \pm 88.9$} & n.s. \\
\hline $\mathrm{T} 3(\mu \mathrm{g} / \mathrm{L})$ & $0.8 \pm 0.2$ & \multicolumn{2}{|c|}{$1.4 \pm 0.4$} & $<0.001$ \\
\hline $\mathrm{T} 4(\mu \mathrm{g} / \mathrm{L})$ & $63.3 \pm 15.8$ & \multicolumn{2}{|c|}{$80.3 \pm 20.8$} & 0.04 \\
\hline \multirow[t]{4}{*}{ TSH (mU/L) } & $1.8 \pm 0.9$ & \multicolumn{2}{|c|}{$1.6 \pm 0.8$} & n.s. \\
\hline & AN High exercisers & AN Low exercisers & Controls & \\
\hline & $n=7$ & $n=5$ & $\mathrm{n}=12$ & \\
\hline & $M \pm S D$ & $M \pm S D$ & $M \pm S D$ & \\
\hline Leptin $(\mu \mathrm{g} / \mathrm{L})$ & $1.6 \pm 1.9$ & $0.9 \pm 1.6$ & $9.6 \pm 4.7$ & $1 c, 2 b$ \\
\hline Cortisol ( $\mu \mathrm{g} / \mathrm{L})$ & $205.7 \pm 65.2$ & $187.1 \pm 41.9$ & $219.6 \pm 88.9$ & \\
\hline T3 $(\mu \mathrm{g} / \mathrm{L})$ & $0.9 \pm 0.3$ & $0.8 \pm 0.2$ & $1.4 \pm 0.4$ & $1 \mathrm{a}, 2 \mathrm{~b}$ \\
\hline $\mathrm{T} 4(\mu \mathrm{g} / \mathrm{L})$ & $59.0 \pm 9.8$ & $71.2 \pm 19.7$ & $80.3 \pm 20.8$ & $1 a$ \\
\hline
\end{tabular}

Values are expressed as means $(\mathrm{M}) \pm$ standard deviations $(\mathrm{SD}) . \mathrm{a}=\mathrm{P}<0.05, \mathrm{~b}=\mathrm{P}<0.01, \mathrm{c}=\mathrm{P}<0.001$, n.s. $=$ not significant.1 $=$ AN high exercisers vs. controls, $2=$ AN low exercisers vs. controls, $3=$ AN low exercisers vs. AN high exercisers. 
Table 4 Psychological data of anorexic patients compared with controls

\begin{tabular}{|c|c|c|c|c|}
\hline \multirow[t]{2}{*}{ Variable } & \multirow{2}{*}{$\begin{array}{l}\text { Anorexia nervosa (AN) } \\
n=12, M \pm S D\end{array}$} & \multirow{2}{*}{\multicolumn{2}{|c|}{$\begin{array}{c}\text { Controls } \\
\mathrm{n}=12, \mathrm{M} \pm \mathrm{SD}\end{array}$}} & \multirow[t]{2}{*}{$P$} \\
\hline & & & & \\
\hline Depression (BDI) & $21.9 \pm 9.6$ & $4.1=$ & & $<0.001$ \\
\hline \multicolumn{5}{|c|}{ Eating disorder inventory (EDI-2) } \\
\hline Body Dissatisfaction & $15.2 \pm 7.3$ & \multicolumn{2}{|c|}{$7.7 \pm 8.7$} & $<0.05$ \\
\hline Social Insecurity & $8.2 \pm 4.1$ & \multicolumn{2}{|c|}{$1.9 \pm 2.3$} & 0.001 \\
\hline Interpersonal Distrust & $5.1 \pm 3.7$ & \multicolumn{2}{|c|}{$0.9 \pm 1.6$} & 0.007 \\
\hline Perfectionism & $6.5 \pm 4.2$ & \multicolumn{2}{|c|}{$2.0 \pm 2.2$} & 0.009 \\
\hline Impulse Regulation & $4.5 \pm 5.3$ & \multicolumn{2}{|c|}{$0.9 \pm 1.7$} & 0.065 \\
\hline Bulimia & $2.8 \pm 5.0$ & \multicolumn{2}{|c|}{$0.3 \pm 0.6$} & 0.027 \\
\hline Interoceptive Awareness & $7.6 \pm 4.6$ & \multicolumn{2}{|c|}{$0.8 \pm 1.4$} & 0.001 \\
\hline Maturity Fears & $4.9 \pm 6.0$ & \multicolumn{2}{|c|}{$1.7 \pm 2.2$} & n.s. \\
\hline Ineffectiveness & $11.8 \pm 8.0$ & \multicolumn{2}{|c|}{$1.2 \pm 1.8$} & 0.002 \\
\hline Ascetism & $7.9 \pm 7.2$ & \multicolumn{2}{|c|}{$1.9 \pm 2.0$} & 0.029 \\
\hline Drive for Thinness & $11.7 \pm 6.0$ & \multicolumn{2}{|c|}{$1.2 \pm 2.3$} & $<0.001$ \\
\hline \multirow[t]{4}{*}{ Eating attitude test (EAT) } & $52.5 \pm 21.0$ & \multicolumn{2}{|c|}{$6.7 \pm 4.1$} & 0.001 \\
\hline & AN High exercisers & AN Low exercisers & Controls & \\
\hline & $\mathrm{n}=7$ & $n=5$ & $\mathrm{n}=12$ & \\
\hline & $M \pm S D$ & $M \pm S D$ & $M \pm S D$ & \\
\hline Drive for thinness (EDI-2) & $14.5 \pm 3.6$ & $9.6 \pm 2.2$ & $1.2 \pm 2.3$ & $1 c, 2 c, 3 a$ \\
\hline Depression (BDI) & $24.3 \pm 9.3$ & $14.6 \pm 5.6$ & $4.1 \pm 2.9$ & $1 c, 2 c, 3 a$ \\
\hline
\end{tabular}

Values are expressed as means $(\mathrm{M}) \pm$ standard deviations $(\mathrm{SD}) . \mathrm{a}=\mathrm{P}<0.05, \mathrm{~b}=\mathrm{P}<0.01, \mathrm{c}=\mathrm{P}<0.001$, n.s. = not significant. $1=\mathrm{AN}$ high exercisers vs. controls, $2=$ AN low exercisers vs. controls, $3=$ AN low exercisers vs. AN high exercisers.

SC item "What percentage of your exercise is aimed at controlling your weight" was significantly correlated with TDEE, we distinguished between low-level and highlevel exercisers according to this item by using a cutoff set at $<50 \%$. There was an increased percentage of highlevel exercisers in patients compared with the controls. This subgroup displayed high TDEE values and also selfreported a considerable amount of daily activity directly related at controlling their weight. The amount of daily activity in low-level AN exercisers appeared to have a decreased amount of daily activity versus the controls, this difference was not statistically significant. Similarly, Bouten et al., 1996 [27] showed that AN patients were more likely to display low or high levels of activity, whereas the controls were more likely to show moderate activity levels.

Interestingly, REE in high-level exercisers was not significantly decreased versus the controls despite the decreased $\mathrm{LBM}, \mathrm{T}_{3}$ and leptin plasma levels. In contrast, low-level AN exercisers displayed lower REE values compared with the high-level AN exercisers and controls, respectively, also when adjusted for LBM and body surface area. However, no differences in thyroid hormone levels were observed between the AN subgroups. In fact, two individuals of the same LBM can differ considerably in their REE due to differences in heredity and a range of physiological factors [34]. The elevated REE in high-level AN patients is possibly caused by a secondary effect resulting from the impact of physical activity on a range of physiological processes that in turn influence REE [34,35]. Additionally it is also conceivable that the body core temperatures within the AN subgroups are slightly different [9].

TDEE was comparable in high-level AN exercisers versus controls, whereas it was reduced in low-level AN exercisers. The PAL in low-level AN exercisers was only 1.16 , whereas in healthy controls and in high-level AN exercisers it was 1.65. Bearing in mind that the low-level AN exercisers and controls had similar activity levels, this reflects the energy-sparing metabolic adaptations to starvation in this AN subgroup. Despite the 3 -fold increased activity levels in high-level AN exercisers compared with the controls, the TDEE values were similar and thus, the energysparing effect, also in this AN subgroup, remarkable. Previous studies have found similar proportions of the PAL in AN patients (not distinguished between low and high activity AN patients) as we did for our high-level AN exercisers [24,27].

Hyperactivity, "drive for thinness" and depression were increased in the $\mathrm{AN}$ patients as previously 
described [5,36]. In this study, this was particularly true for the high-level exercisers, a finding which is supported by the study of Penas-Lledo et al., 2002 [37] where data on depression were retrieved from SCL-90-R scores. Consequently, this group compensated their "drive for thinness" by an increased amount of daily activity. This hyperactivity may be a component of an obsessive-compulsive behavior but could also serve to improve mood and manage stress, thus regulating negative emotions or affects $[38,39]$. Several studies have shown that exercise has a positive impact on a variety of psychological disorders $[40,41]$. Nevertheless, the increased amount of daily activity in the high-level AN exercisers was not sufficient to suppress their levels of depression to values of low-level AN exercisers or controls. In addition to the aspect and function of comorbid depression in association with pattern of high-level exercise as well as an association with binge/purge behaviour additional aspects e.g. level of impulsivity, might help to characterize AN patients to identify more homogenous endophenotypes of AN patients [42]. From an evolutionary point of view, this hyperactivity could be a result of food search behavior [43]. If in the long-term this is not rewarded by the intake of a substantial amount of food, this phenomenon could explain the increased observed depression in this subgroup.

We and others [24] did not find elevated plasma cortisol levels, although hypercortisolemia has been described in AN and in depressive patients [44]. This may be due to marked interindividual differences in cortisol levels, intraindividual stability across the day, and a range of other factors that may affect cortisol levels including physical exercise, sleep, exhaustion/fatigue, anxiety/negative effects, work hours and stress $[45,46]$.

\section{Limitations of the study}

The habits on activity were assessed by applying a structured interview (DDE) and not measured directly using a technique like the triaxial accelerometry. Thus, our study can not distinguish between different components of high energy expenditure e.g. high levels of exercise or micromovements. As in most studies using the doubly labeled water technique, our study population was rather small. Therefore, prospective studies with larger AN samples using both validated measures of self-reported and objectively assessed physical activity (e.g. accelerometry) combined with psychobiological aspects of starvation and reward processing might be an interesting perspective to elucidate the important aspect of hyperactivity in AN patients.

\section{Conclusions}

Taken together, our study shows that there are marked differences in energy requirement among AN patients. Over one-half of our AN patients reported high exercise levels directly related to weight control with an overrepresentation of the binge/purge AN subgroup. Bearing in mind their dramatically increased energy expenditure and levels of depression, this subgroup may benefit from a structured treatment program specifically addressing their hyperactivity [47] supplemented where necessary with drugs derived from the group of atypical antipsychotic [48]. Studies thus far with olanzapine in AN patients have shown some promise with respect to weight gain and improvement of depression, anxiety, aggressiveness, obsessive-compulsiveness and also safety [48-50].

In order to assess the actual energy requirements and more appropriately match treatment to patients needs in $\mathrm{AN}$, it is important for clinicians to quantify both energy intake and physical exercise levels. A simple and expedient way to ascertain the latter before and during treatment would be the examination of the single item "What percentage of your exercise is aimed at controlling your weight" of the EDI-SC.

\section{Competing interest}

There is no conflict of interest in this paper for any of the authors.

\section{Authors' contributions}

All authors: read and approved the final manuscript. SZ: conception, design and performance of the study, acquisition of data, statistical data analysis and interpretation of data, drafted the paper. IM: interpretation of data, drafted the paper. LAB: interpretation of data. JH: laboratory analysis, interpretation of data. ST: conception and design of the study. WH: interpretation of data. SA: performance of the study. BSWD: laboratory analysis, revised the paper critically for important intellectual content. JR: conception and design of the study, revised the paper critically for important intellectual content.

\section{Acknowledgements}

We thank particularly the late Prof. Pierre JV Beumont, (MB ChB MPhil Oxford), Department of Psychological Medicine, University of Sydney, Australia for supporting this study. In addition this study was supported by a grant from the Deutsche Forschungsgemeinschaft (S.Z.) and a competitive project grant from the Australian National Health and Medical Research Council (\#95-0250). The study was performed at the Northside Clinic Greenwich NSW 2065, Australia, a Ramsay Healthcare hospital and this organization provided additional research support.

\section{Author details}

${ }^{1}$ Department of Psychosomatic Medicine \& Psychotherapy, University Medical Hospital Tuebingen, Osianderstr. 5, 72074 Tuebingen, Germany. ${ }^{2}$ Department of Psychological Medicine, University of Sydney, Sydney, Australia. ${ }^{3}$ Department of Paediatrics \& Child Health, University of Sydney, Sydney, Australia. ${ }^{4}$ School of Psychology and Discipline of Psychiatry, University of Sydney, Sydney, Australia. ${ }^{5}$ Department of Obstetrics \& Gynaecology, University of Sydney, Sydney, Australia. 'Eating Disorders Unit, Northside Clinic, Greenwich NSW 2065, Australia. ${ }^{7}$ Department of General Internal and Psychosomatic Medicine, University of Heidelberg, Heidelberg, Germany. ${ }^{8}$ Department of Child \& Adolescent Psychiatry, Univ. of Essen, Essen, Germany. ${ }^{9}$ Children's Nutrition Research Centre, School of Medicine, University of Queensland, Brisbane, Australia. 
Received: 6 June 2013 Accepted: 27 August 2013

Published: 4 September 2013

\section{References}

1. Zipfel S, Lowe B, Reas DL, Deter HC, Herzog W: Long-term prognosis in anorexia nervosa: lessons from a 21-year follow-up study. Lancet 2000, 355(9205):721-722.

2. Lasegue E: De l'anorexie hystérique. Archives Générales de Médicine 1873, 1:385-403.

3. Gull W: Anorexia nervosa (apepsia hysterica, anorexia hysterica). Clin Soc Trans 1874, 7:22-28.

4. Casper RC: The 'drive for activity' and "restlessness" in anorexia nervosa: potential pathways. J Affect Disord 2006, 92(1):99-107.

5. Dalle Grave R, Calugi S, Marchesini G: Compulsive exercise to control shape or weight in eating disorders: prevalence, associated features, and treatment outcome. Compr Psychiatry 2008, 49(4):346-352.

6. Valanou EM, Bamia C, Trichopoulou A: Methodology of physical-activity and energy-expenditure assessment: a review. J Public Health 2006, 14:58-65.

7. Landsberg L, Young JB, Leonard WR, Linsenmeier RA, Turek FW: Do the obese have lower body temperatures? A new look at a forgotten variable in energy balance. Trans Am Clin Climatol Assoc 2009, 120:287-295.

8. Schoeller DA, Leitch CA, Brown C: Doubly labeled water method: in vivo oxygen and hydrogen isotope fractionation. Am J Physiol 1986, 251(6 Pt 2):R1137-R1143.

9. Landsberg L, Young JB, Leonard WR, Linsenmeier RA, Turek FW: Is obesity associated with lower body temperatures? Core temperature: a forgotten variable in energy balance. Metabolism 2009, 58(6):871-876.

10. Barbosa-Saldivar JL, Van Itallie TB: Semistarvation: an overview of an old problem. Bull N Y Acad Med 1979, 55(8):774-797

11. Misra M, Klibanski A: Neuroendocrine consequences of anorexia nervosa in adolescents. Endocr Dev 2010, 17:197-214.

12. de Zwaan M, Aslam Z, Mitchell JE: Research on energy expenditure in individuals with eating disorders: a review. Int J Eat Disord 2002, 32(2):127-134

13. Russell J, Baur LA, Beumont PJ, Byrnes S, Gross G, Touyz S, Abraham S, Zipfel $\mathrm{S}$ : Altered energy metabolism in anorexia nervosa. Psychoneuroendocrinology 2001, 26(1):51-63.

14. Russell J, Baur L, Beumont $P$, Byrnes $S$, Zipfel S: Refeeding of anorexics: wasteful not wilful. Lancet 1998, 352(9138):1445-1446.

15. Heilbronn LK, Millner K-L, Kriketos A, Russell J, Campbell LV: Metabolic dysfunction in anorexia nervosa. Obes Res Clin Pract 2007, 1:139-146.

16. Gross G: Longitudinal study of patients with anorexia nervosa 6 to 10 years after treatment. Hamburg: Verlag Kovac; 2002. ISBN ISBN 3-8300-0498-2.

17. Beck AT, Epstein N, Brown G, Steer RA: An inventory for measuring clinical anxiety: psychometric properties. J Consult Clin Psychol 1988, 56(6):893-897.

18. Garner DM: The Eating Disorder Inventory 2. Odessa, FL: Psychological Assessment Resources; 1991.

19. Garner DM, Garfinkel PE: The Eating Attitudes Test: an index of the symptoms of anorexia nervosa. Psychol Med 1979, 9(2):273-279.

20. Durnin JV, Womersley J: Body fat assessed from total body density and its estimation from skinfold thickness: measurements on 481 men and women aged from 16 to 72 years. Br J Nutr 1974, 32(1):77-97.

21. Coward WA: The doubly-labelled water method: principles and practice. Proc Nutr Soc 1988, 47:209-218.

22. Davies PSW, Ewing G, Lucas A: Energy expenditure in early infancy. Br J Nutrition 1989, 62:621-629.

23. Platte $P$, Pirke KM, Trimborn P, Pietsch K, Krieg JC, Fichter MM: Resting metabolic rate and total energy expenditure in acute and weight recovered patients with anorexia nervosa and in healthy young women. Int J Eat Disord 1994, 16(1):45-52.

24. Casper RC, Schoeller DA, Kushner R, Hnilicka J, Gold ST: Total daily energy expenditure and activity level in anorexia nervosa. Am J Clin Nutr 1991, 53(5):1143-1150.

25. Pirke KM, Trimborn P, Platte P, Fichter M: Average total energy expenditure in anorexia nervosa, bulimia nervosa, and healthy young women. Biol Psychiatry 1991, 30(7):711-718.
26. Bossu C, Galusca B, Normand S, Germain N, Collet P, Frere D, Lang F, Laville M, Estour B: Energy expenditure adjusted for body composition differentiates constitutional thinness from both normal subjects and anorexia nervosa. Am J Physiol Endocrinol Metab 2007, 292(1):E132-E137.

27. Bouten CV, van Marken Lichtenbelt WD, Westerterp KR: Body mass index and daily physical activity in anorexia nervosa. Med Sci Sports Exerc 1996 28(8):967-973.

28. van Marken Lichtenbelt WD, Heidendal GA, Westerterp KR: Energy expenditure and physical activity in relation to bone mineral density in women with anorexia nervosa. Eur J Clin Nutr 1997, 51(12):826-830.

29. Melchior JC, Rigaud D, Rozen R, Malon D, Apfelbaum M: Energy expenditure economy induced by decrease in lean body mass in anorexia nervosa. Eur J Clin Nutr 1989, 43(11):793-799.

30. Onur S, Haas V, Bosy-Westphal A, Hauer M, Paul T, Nutzinger D, Klein H, Muller MJ: L-tri-iodothyronine is a major determinant of resting energy expenditure in underweight patients with anorexia nervosa and during weight gain. Eur J Endocrinol 2005, 152(2):179-184.

31. Holtkamp K, Herpertz-Dahlmann B, Mika C, Heer M, Heussen N, Fichter M, Herpertz S, Senf W, Blum WF, Schweiger U, Warnke A, Ballauff A, Remschmidt H, Hebebrand J: Elevated physical activity and low leptin levels co-occur in patients with anorexia nervosa. J Clin Endocrinol Metab 2003, 88(11):5169-5174.

32. Holtkamp K, Herpertz-Dahlmann B, Hebebrand K, Mika C, Kratzsch J, Hebebrand J: Physical activity and restlessness correlate with leptin levels in patients with adolescent anorexia nervosa. Biol Psychiatry 2006, 60(3):311-313

33. Nogueira JP, Maraninchi M, Lorec AM, Corroller AB, Nicolay A, Gaudart J, Portugal H, Barone R, Vialettes B, Valero R: Specific adipocytokines profiles in patients with hyperactive and/or binge/purge form of anorexia nervosa. Eur J Clin Nutr 2011, 64(8):840-844

34. Speakman JR, Selman C: Physical activity and resting metabolic rate. Proc Nutr Soc 2003, 62(3):621-634.

35. LaForgia J, Withers RT, Gore CJ: Effects of exercise intensity and duration on the excess post-exercise oxygen consumption. J Sports Sci 2006, 24(12):1247-1264.

36. O'Brien KM, Vincent NK: Psychiatric comorbidity in anorexia and bulimia nervosa: nature, prevalence, and causal relationships. Clin Psychol Rev 2003, 23(1):57-74.

37. Penas-Lledo E, Vaz Leal FJ, Waller G: Excessive exercise in anorexia nervosa and bulimia nervosa: relation to eating characteristics and general psychopathology. Int J Eat Disord 2002, 31(4):370-375.

38. Davis C, Kaptein S: Anorexia nervosa with excessive exercise: phenotype with close links to obsessive-compulsive disorder. Psychiatry Res 2006, 142(2-3):209-217.

39. Vansteelandt K, Pieters G, Vandereycken W, Claes L, Probst M, Van Mechelen I: Hyperactivity in anorexia nervosa: a case study using experience sampling methodology. Eat Behav 2004, 5(1):67-74.

40. Strohle A: Physical activity, exercise, depression and anxiety disorders. J Neural Transm 2009, 116(6):777-784.

41. Kanning $M$, Schlicht $W$ : Be active and become happy: an ecological momentary assessment of physical activity and mood. J Sport Exerc Psychol 2010, 32(2):253-261.

42. Penas-Lledo E, Fernandez-Aranda F, Jimenez-Murcia S, Granero R, Penelo E, Soto A, Gunnard K, Menchon JM: Subtyping eating disordered patients along drive for thinness and depression. Behav Res Ther 2009, 47(6):513-519.

43. Sodersten P, Nergardh R, Bergh C, Zandian M, Scheurink A: Behavioral neuroendocrinology and treatment of anorexia nervosa. Front Neuroendocrinol 2008, 29(4):445-462.

44. Wolkowitz OM, Burke H, Epel ES, Reus VI: Glucocorticoids. Mood, memory, and mechanisms. Ann N Y Acad Sci 2009, 1179:19-40.

45. Almeida DM, Piazza JR, Stawski RS: Interindividual differences and intraindividual variability in the cortisol awakening response: an examination of age and gender. Psychol Aging 2009, 24(4):819-827.

46. Dahlgren A, Kecklund G, Theorell T, Akerstedt T: Day-to-day variation in saliva cortisol-relation with sleep, stress and self-rated health. Biol Psychol 2009, 82(2):149-155.

47. Beumont PJ, Arthur B, Russell JD, Touyz SW: Excessive physical activity in dieting disorder patients: proposals for a supervised exercise program. Int J Eat Disord 1994, 15(1):21-36. 
48. Flament MF, Bissada H, Spettigue W: Evidence-based pharmacotherapy of eating disorders. Int I Neuropsychopharmacol 2011, 15(2):189-207.

49. Attia E, Kaplan AS, Walsh BT, Gershkovich M, Yilmaz Z, Musante D, Wang Y: Olanzapine versus placebo for out-patients with anorexia nervosa. Psychol Med 2011, 41(10):2177-2182.

50. McKnight RF, Park RJ: Atypical antipsychotics and anorexia nervosa: a review. Eur Eat Disord Rev 2010, 18(1):10-21.

doi:10.1186/2050-2974-1-37

Cite this article as: Zipfel et al:: Impact of exercise on energy

metabolism in anorexia nervosa. Journal of Eating Disorders 2013 1:37.

\section{Submit your next manuscript to BioMed Central} and take full advantage of:

- Convenient online submission

- Thorough peer review

- No space constraints or color figure charges

- Immediate publication on acceptance

- Inclusion in PubMed, CAS, Scopus and Google Scholar

- Research which is freely available for redistribution 